\title{
Характеристики надежности современных ПЛИС
}

\author{
А. Строгонов, д. Т. н. ${ }^{1}$
}

УДК 621.3.049.774 | ВАК 05.27.01

Сегодня ПлиС все чаще применяются в аппаратуре ответственного назначения , которая должна отвечать высоким требованиям по надежности. Однако непрерывное уменьшение технологических проектных норм при изготовлении интегральных схем усугубляет проблему обеспечения отказоустойчивости высокоинтегрированных ПЛИС. Анализ интенсивности отказов и прогнозирование характеристик надежности ПлиС проводят по результатам ускоренных испытаний, которые за относительно короткий период времени позволяют оценить вероятность появления отказов в процессе эксплуатации устройства. Рассмотрим методику расчета экспериментальной интенсивности отказов ПЛиС с использованием статистики хи-квадрат, а также влияние сбоев, вызванных ионизирующим излучением, на характеристики надежности современных ПЛис, выпускаемых по нанометровым проектным нормам.

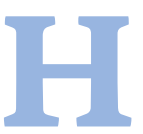

адежность БИс, в том числе плис, при эксплуатации характеризуется известной кривой зависимости интенсивности отказов от времени. Эта кривая показывает относительно высокую интенсивность отказов на ранней стадии (в период приработки), сравнительно низкую и стабильную интенсивность отказов в период эксплуатации и возрастающую интенсивность в период износа (после примерно 25 лет непрерывной работы) [1, 2]

Интенсивность отказов зарубежных ПЛИС принято выражать в ФИТах (FIT - Failure in Time, или число отказов за полное время испытаний), так как это удобно и наглядно отражает сверхмалые интенсивности отказов: ФИТ $=\lambda \cdot 10^{9}$. Интенсивность отказов $\lambda$ характеризуется размерностью 1/ч. Поэтому, например, 1 ФИТ эквивалентен одному отказу на 109 приборов в час, интенсивность отказов 5 ФИТ означает, что прогнозируется пять отказов на 1 млн ИС, имеющих наработку 10004.

Вероятность безотказной работы Р(t) для экспоненциального закона распределения вероятностей отказа случайной величины для периода нормальной работы равна [3-5]:

$$
P(t)=e^{-\lambda t}=e^{-\frac{t}{t_{0}}}
$$

где $t_{0}=M T T F-$ среднее время наработки до первого отказа или ее математическое ожидание:

Воронежский государственный технический университет, профессор кафедры полупроводниковой электроники и наноэлектроники, тел. +7 4732 43-76-95, andreistrogonov@mail.ru.

$$
\operatorname{MTTF}=1 / \lambda
$$

Интенсивность отказов может быть оценена по формуле (3) в предположении, что действуют механизмы отказа, выявляемые температурой [3-5]:

$$
\lambda=\frac{\Delta n}{N \Delta t K_{y}^{T}},
$$

где $\Delta n-$ число отказавших ИС за время испытаний,

$\mathrm{N}$ - число ИС в выборке,

$\Delta t$ - длительность испытаний,

$\mathrm{K}_{y}^{\mathrm{T}}$ - коэффициент ускорения отказов, выявляемых температурой.

$\mathrm{K}_{y}^{\mathrm{T}}$ показывает экспоненциальную зависимость от температуры в диапазоне 0,3-1,3 эВ. Если принять, что различные дефекты влияют примерно одинаково на изменение интенсивности отказов, то среднюю энергию активации можно считать равной 0,4 эВ. В соответствии с правилом «10 градусов» принимают, что скорость старения увеличивается вдвое при повышении температуры на $10^{\circ} \mathrm{C}$. Этому закону нарастания скорости старения соответствует энергия активации в пределах 0,7-0,8 эВ. Поэтому величину 0,7 эВ обычно используют в расчетах интенсивности отказов.

В качестве показателя снижения долговечности прибора в результате развития дефектов при повышении температуры от $\mathrm{T}_{1}$ до $\mathrm{T}_{2}$ используют коэффициент ускорения $K_{y}^{\mathrm{T}}[1-5]$ :

$$
\mathrm{K}_{y}^{\mathrm{T}}=\frac{t_{1}}{t_{2}}=\exp \left(\frac{E_{a}}{k}\left[\frac{1}{\mathrm{~T}_{1}}-\frac{1}{\mathrm{~T}_{2}}\right]\right),
$$


или с учетом рассеиваемой мощности на переходе:

$$
K_{y}^{\mathrm{T}}=\frac{t_{1}}{t_{2}}=\exp \left(\frac{E_{a}}{k}\left[\frac{1}{T_{1}+\left(P_{1} \cdot \theta_{1}\right)}-\frac{1}{T_{2}+\left(P_{2} \cdot \theta_{2}\right)}\right]\right)
$$

где верхний индекс Т отражает влияние температуры; $t$ $n t_{2}$ - среднее время наработки на отказ при температуре $\mathrm{T}_{1}$ и $\mathrm{T}_{2}$ соответственно; $\mathrm{T}_{1}, \mathrm{~T}_{2}$ - нормальная и повышенная температура соответственно (например, $\left.\mathrm{T}_{1}\left({ }^{\circ} \mathrm{K}\right)=55^{\circ} \mathrm{C}+273, \mathrm{~T}_{2}\left({ }^{\circ} \mathrm{K}\right)=125^{\circ} \mathrm{C}+273\right) ; \mathrm{k}=8,167 \cdot 10^{-5}$ эВ $/{ }^{\circ} \mathrm{K}$; $\mathrm{E}_{a}$ - энергия активации для наблюдаемого процесса старения; $\mathrm{P}_{1}, \mathrm{P}_{2}$ - рассеиваемая мощность на переходе; $\theta_{1}, \theta_{2}$ - термические коэффициенты сопротивления (ТКС) «переход - окружающая среда».

На рис. 1 показана экстраполяция результатов ускоренных испытаний, проводимых при температуре $167,5^{\circ} \mathrm{C}$, на условия эксплуатации при температуре $70{ }^{\circ} \mathrm{C} п р и$ $\mathrm{K}_{y}^{\mathrm{T}}=188$ и $\mathrm{E}_{a}=0,7$ эВ [6].

Если в качестве ускоряющего фактора использовать напряженность электрического поля, то для выявления дефектов оксида коэффициент ускорения равен [1, 7-9]:

$$
K_{y}^{U}=\exp \left(\beta \Delta U_{n}\right)
$$

где $\Delta \mathrm{U}_{n}=\mathrm{U}_{1}-\mathrm{U}_{2}, \mathrm{U}_{2}<\mathrm{U}_{1}$ (для различных серий ПлИС используются разные напряжения), $\mathrm{U}_{2}$ - повышенное напряжение при испытаниях, $U_{1}$ - рабочее напряжение, например, 13,2 и 12 В или 3,96 и 3,3 В.
Зарубежные компании-производители плис в качестве моделей коэффициента ускорения (ускоряющий фактор - повышенное напряжение) используют следующие выражения:

- Atmel [7]: $K_{y}^{U}=\exp \left(\beta \Delta U_{n}\right)$, где $\beta=0,5-1\left[B^{-1}\right]$;

- Altera $[8,9]$ : для подзатворных диэлектриков МОП-транзисторов $-K_{\text {уд }}=\exp \left(\beta / h_{\text {д }} / 10 n m \Delta U_{n}\right)$, где $h_{\text {д }}$ толщина подзатворного диэлектрика МОП-транзистора (при технологических проектных нормах более 90 нм, $E_{a}=0,7$ эВ, $\beta=3,2\left[\mathrm{~B}^{-1}\right]$ ); для межслойных диэлектриков металлизации $\left(E_{a}=0,7 \ni B, \beta=2,0\left[B^{-1}\right]\right)-K_{\text {умд }}=\exp \left(\beta \Delta U_{n}\right)$.

Экспериментальную интенсивность отказов Плис ведущие компании (в частности Atmel, Xilinx, Altera, QuickLogic, Actel) оценивают с использованием статистики Х2 (хи-квадрат) [1-11]:

$$
\lambda=\frac{\chi^{2}\left(\mathrm{P}^{\prime \prime}, \mathrm{m}\right)}{2 \mathrm{~N} \Delta t \mathrm{~K}_{\mathrm{y}}} \cdot 10^{9}=\frac{\mathrm{U}}{\mathrm{N} \Delta t \mathrm{~K}_{\mathrm{y}}} \cdot 10^{9},
$$

где $\mathrm{U}=\frac{\chi^{2}\left(\mathrm{P}^{*}, \mathrm{~m}\right)}{2}, \mathrm{X}^{2}$ - распределение хи-квадрат (табулированная величина, зависящая от доверительной вероятности и числа отказов); Р" - доверительная вероятность, связанная с уровнем значимости CL соотношением $\mathrm{P}^{*}=1-\frac{\mathrm{CL} \%}{100} ; m=(2 n+2)-$ число степеней свободы, где $n-$ количество отказавших ИС; $\mathrm{N}$ - общее число испытуемых ИС; $K_{y}$ - обобщенный коэффициент ускорения

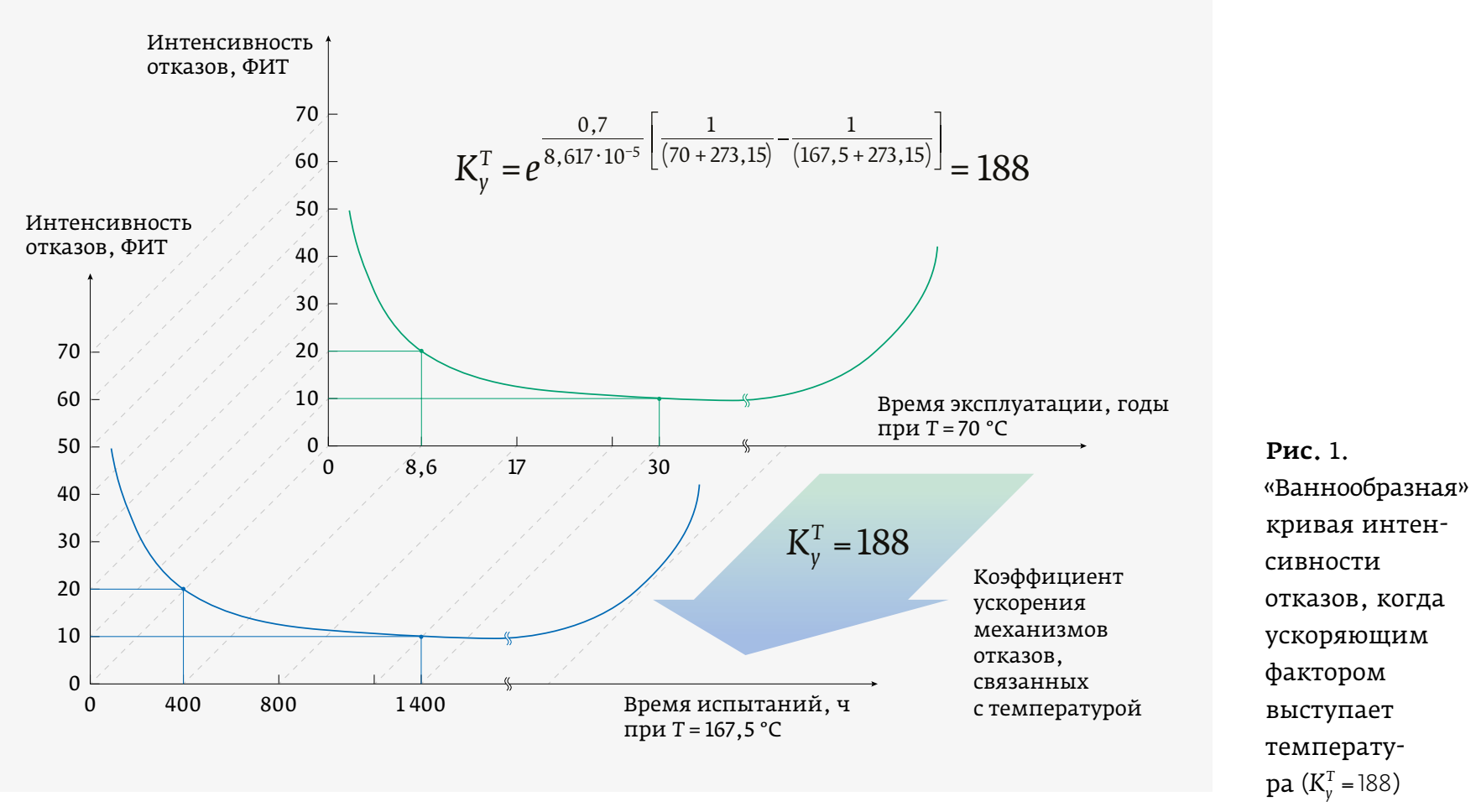


Таблица 1. Интенсивность отказов с использованием статистики $\chi^{2}$ с 60\%-ным уровнем значимости по результатам ускоренных испытаний КМОП ПлиС Microsemi

\begin{tabular}{|c|c|c|c|c|}
\hline Технология, тип Плис & $\begin{array}{c}\text { Число } \\
\text { отказов }\end{array}$ & $\begin{array}{l}\text { Приборо- } \\
\text { часы }\end{array}$ & ФИТ & MTTF \\
\hline 1,0 мкм, КМОП СОзУ пЛис & 1 & $3,66 \cdot 10^{8}$ & 5,53 & $1,81 \cdot 10^{8}$ \\
\hline 1,0 мкм, КМОП СОзУ плис (RH1020) & 0 & $3,97 \cdot 10^{7}$ & 23,05 & $4,34 \cdot 10^{7}$ \\
\hline 0,8 мкм, КМОП ПЛИС (RH1280) & 1 & $9,16 \cdot 10^{7}$ & 22,04 & $4,54 \cdot 10^{7}$ \\
\hline 0,6 мкм, КМОП СОЗУ (RT54SX) & 0 & $2,29 \cdot 10^{7}$ & 39,98 & $2,51 \cdot 10^{7}$ \\
\hline 0,45 мкм, UМС КМОП СОзУ Плис & 0 & $4,50 \cdot 10^{7}$ & 20,32 & $4,92 \cdot 10^{7}$ \\
\hline 0,25 мКМ, МЕС КМОП СОЗУ ПЛИС & 2 & $7,51 \cdot 10^{7}$ & 41,40 & $2,42 \cdot 10^{7}$ \\
\hline 0,25 мкм, UМС КМОП СОзУ Плис & 0 & $7,93 \cdot 10^{8}$ & 1,15 & $8,66 \cdot 10^{8}$ \\
\hline 0,15 мкм, UМС КМОП Созу Плис & 2 & $5,24 \cdot 10^{8}$ & 5,93 & $1,69 \cdot 10^{8}$ \\
\hline 0,13 мкм, UMC КМОП флеш-Плис & 1 & $2,89 \cdot 10^{8}$ & 7,00 & $1,43 \cdot 10^{8}$ \\
\hline 65 нм, UMC КМОП флеш-Плис & 0 & $2,95 \cdot 10^{8}$ & 3,11 & $3,22 \cdot 10^{8}$ \\
\hline
\end{tabular}

Рассмотрим порядок расчета интенсивности отказов, когда при испытаниях используются два ускоряющих фактора: повышенная температура и повышенное напряжение питания [3]. За рубежом используют понятие HTOL - High Temperature Operating Life Test (метод 1005, 1006 по MIL-STD - 883). В отечественной практике под HTOL понимают электротермотренировку (ЭТТ), статическую или динамическую, которая проводится при предельных электрических нагрузках (обычно 20-30\% номинального напряжения питания $\left.V_{c c}\right)$ и предельной температуре (125-150 C). Время испытаний не превышает 1000 ч. ЭТТ ускоряет процесс старения БИС (в том числе Плис) и предназначена для "выжигания" ранних отказов и повышения надежности

(например, $K_{y}=K_{y}^{\mathrm{T}} K_{y}^{U}$ ); $\Delta t$ - время испытаний; $N \Delta t K_{y}-п р и-$ веденное полное время испытаний или эквивалентные приборо-часы (в зарубежной литературе принята аббревиатура EDH - Equivalent Device Hours). Значения EDH обычно приводятся к температуре $55^{\circ} \mathrm{C}$.

Использование формулы (7) предполагает рассмотрение значения $U$ как случайной величины, распределенной по закону $\chi^{2}[6,8,9]$. При отсутствии отказов за время испытаний $(n=0, m=2)$ их интенсивность описывается экспоненциальным распределением времени до наступления отказа. Оценка интенсивности отказов Плис, полученная с использованием распределения $\chi^{2}$, справедлива только для периода нормальной работы устройства на кривой интенсивности отказов.

Таблица 2. Интенсивность отказов с использованием статистики $\chi^{2}$ с 60\%-ным уровнем значимости по результатам ускоренных испытаний плис Altera

\begin{tabular}{lcccc} 
Серия Плис & $\begin{array}{c}\text { Техноло- } \\
\text { гия, нм }\end{array}$ & $\begin{array}{c}\text { Приборо- } \\
\text { часы }\end{array}$ & $\begin{array}{c}\text { Число } \\
\text { отказов }\end{array}$ & $\begin{array}{c}\text { Интенсивность } \\
\text { отказов, ФИТ } \\
\text { (при CL=60\%) }\end{array}$ \\
\hline Arria 10 (2017 год) & 20 & 685500 & 0 & 52,1 \\
\hline Stratix V (2014 год) & 28 & 628714 & 0 & 47,0 \\
\hline Cyclone V (2014 год) & 28 & 1138666 & 0 & 24,5 \\
\hline Stratix IV (2014 год) & 40 & 1222311 & 2 & 42,0 \\
\hline Stratix III (2014 год) & 65 & 1947828 & 0 & 11,1 \\
\hline FLEX10КА (2014 год) & $300 / 350$ & 330000 & 0 & 53,7 \\
\hline
\end{tabular}

оставшихся в партии изделий. Динамическая ЭТТ связана с отказами в результате износа с низкой энергией активации (порядка 0,3-0,4 эв).

Условия и режимы проведения ЭТТ для определения интенсивности отказов зарубежные компании устанавливают самостоятельно, однако в большинстве случаев они аналогичны указанным в MIL-HDBK-217.

Результаты ускоренных испытаний КМОП плИС типа FPGA компании Microsemi с 60\%-ным уровнем значимости и энергией активации отказов $\mathrm{E}_{a}=0,7$ эВ, приведенные к температуре $55^{\circ} \mathrm{C}$, представлены в табл. 1 [11]. А в табл. 2 отражены результаты ускоренных испытаний КМОП ПЛИС типа FPGA компании Altera с 60\%-ным уровнем значимости и энергией активации отказов 0,7 эв, приведенные к температуpe $70^{\circ} \mathrm{C}$.

Доминирующими механизмами отказов для ПлИС серии Stratix $V$, изготовленных по 28-нм КМОПпроцессу (High Performance) кремниевой фабрики TSMC являются: пробой подзатворного диэлектрика (0,53 эв); пробой межслойных диэлектриков (0,56 эв); сквозные пустоты (1 эВ). Серия Stratix $V$ работает при напряжениях питания $V_{c c}$ 0,85 или 0,9 В в зависимости от скорости ПлИС. При ускоренных испытаниях использовались напряжения 1,1 и 1,2 В. Общая интенсивность отказов с применением 


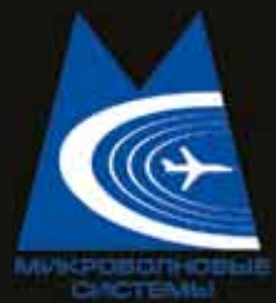

ИНТЕЛЛЕКТ. КАЧЕСТВО.

АО «МИКРОВОЛНОВЫЕ СИСТЕМЫ»

Москва, ул. Нижняя Сыромятническая, 11

Teл. (495) 917-21-03

Факс (495) 917-19-70

E-mail:mwsystems@mwsystems.ru

www.mwsystems.ru

- СОВРЕМЕННОЕ ПРОИЗВОДСТВО И ТЕХНОЛОГИИ

- OПТИМАЛЬHОЕ СООТНОШЕНИЕ ЦLEHA/КАЧЕСТВO

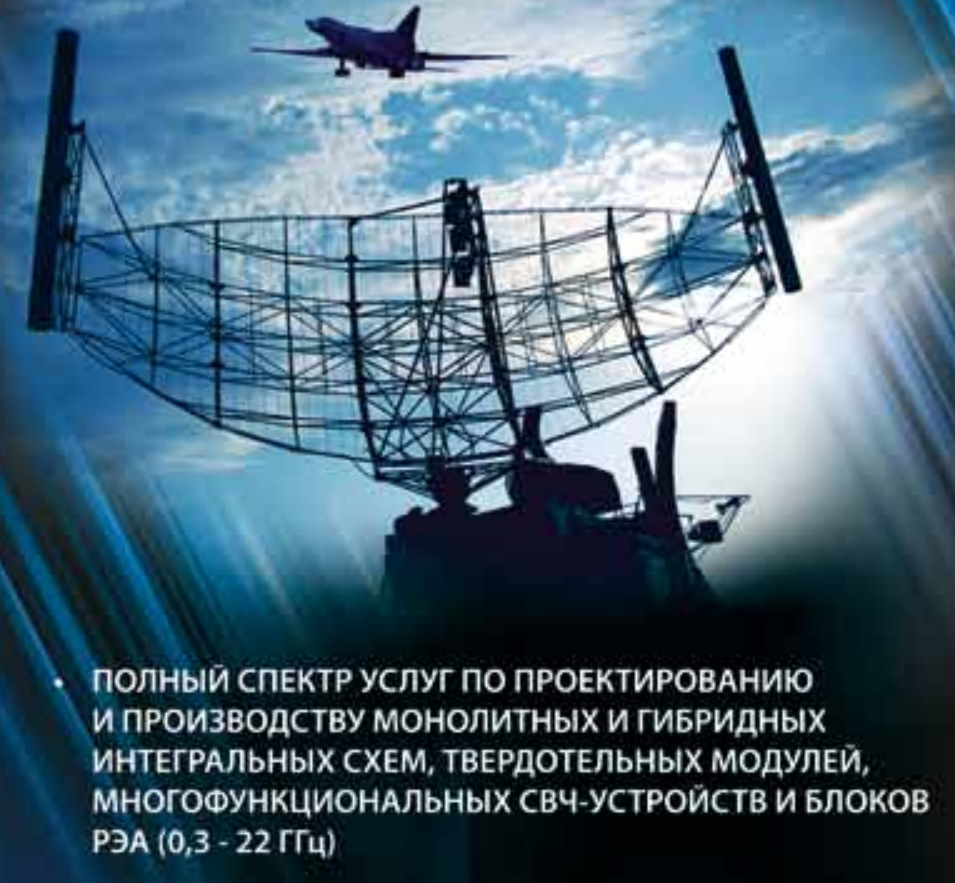

\section{АКЦИОНЕРНОЕ ОБЩЕСТВО «МИКРОВОЛНОВЫЕ СИСТЕМЫ»}
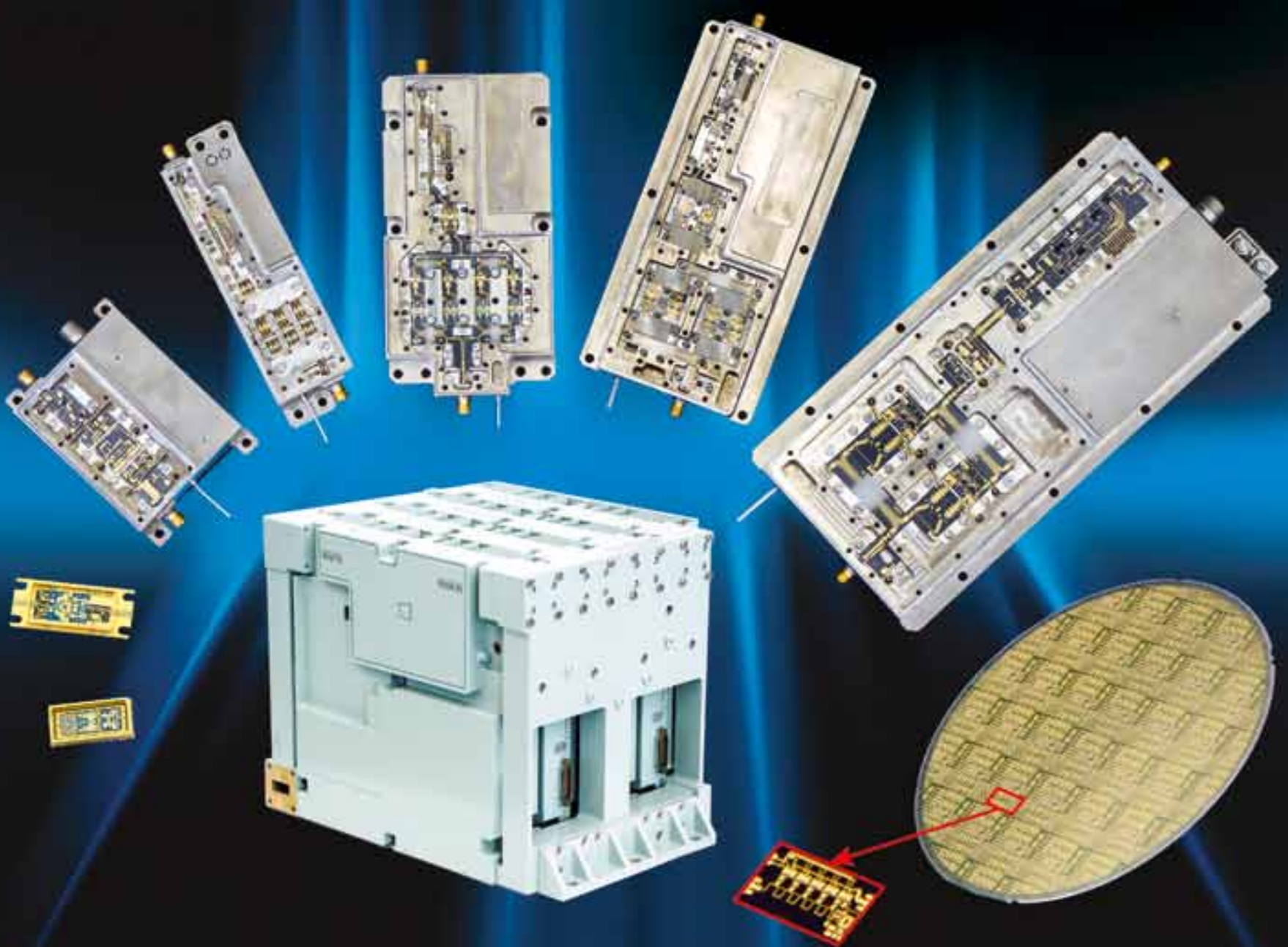
Таблица 3. Интенсивность отказов с использованием статистики $\chi^{2}$ с 60\%-ным уровнем значимости по результатам ускоренных испытаний Плис Xilinx

\begin{tabular}{|c|c|c|}
\hline $\begin{array}{l}\text { Технология, } \\
\text { нм / серия }\end{array}$ & $\begin{array}{c}\text { Приборо-часы } \\
\text { при температуре } \\
\text { испытаний } 125^{\circ} \mathrm{C} \\
\text { (годы выпуска } 2018 / 2019 \text { ) }\end{array}$ & $\begin{array}{c}\text { Интенсивность } \\
\text { отказов, ФИТ } \\
\text { (при CL=60\%, годы } \\
\text { выпуска 2018/2019) }\end{array}$ \\
\hline 16/UltraScale+ & $1496698 / 1411811$ & $8 / 10$ \\
\hline 20/UltraScale & $1016149 / 1054884$ & $11 / 13$ \\
\hline $\begin{array}{l}28 \text { / Плис 7-й серии } \\
\text { (включая Zynq-7000) }\end{array}$ & $1051651 / 1133597$ & $11 / 11$ \\
\hline $45 / X C 6 S x x x$ & $1036231 / 1027456$ & $11 / 6$ \\
\hline $\begin{array}{l}\text { 65/XC5VxXxxx, } \\
\text { XCE5VxXxxx }\end{array}$ & $1808112 / 2036838$ & $6 / 2$ \\
\hline $180 / \mathrm{XC} 2 \mathrm{CXxx}$ & $1086123 / 1086123$ & $11 / 11$ \\
\hline
\end{tabular}

играть проблема сбоев, вызванных ионизирующим излучением. Эти виды отказов можно сравнить с такими проблемами, снижающими надежность современных БИС, как электромиграция, времязависимый пробой подзатворного диэлектрика и др.

Современные плис, выпускаемые по нанометровым проектным нормам, как на основе созу или флеш-памяти, так и с токопроводящими перемычками (antifuse), в той или иной мере подвержены радиационным воздействиям, что может ограничивать их применение в будущем [14]. Одиночные сбои переключения ячеек памяти, проявляющиеся в обратимом изменении ее состояния, вызванные ионизирующим излучением (Single

статистики $\chi^{2}$ при нуле отказов составила 47 ФИТ. Согласно отчету за I квартал 2017 года интенсивность отказов ИС этой же серии, изготовленной в TSMC по аналогичному техпроцессу, составила 38,5 ФИТ [8, 9].

Результаты ускоренных испытаний КМОП ПлИс от Xilinx с 60\%-ным уровнем значимости и энергией активации отказов 0,7 эВ, приведенные к температуре $55^{\circ} \mathrm{C}$, представлены в табл. $3[12,13]$.

При переходе на новые технологические поколения плис по мере уменьшения геометрических размеров транзисторов и напряжения питания важную роль стала
Event Upset - SEU), рассматривались раньше как проблема, актуальная только для аэрокосмических вариантов применения. Однако за последние годы выяснилось, что эта проблема распространилась и на БИС, работающие в атмосфере и даже на уровне Земли, стала одним из основных источников системных сбоев радиоаппаратуры.

Рассмотрим ситуацию на примере Плис компании Xilinx. В табл. 4 представлены сведения об интенсивности программных ошибок (которые принято измерять

Таблица 4. Интенсивность программных ошибок $\lambda$ для Плис Xilinx разных технологических поколений при экспериментальных испытаниях и в условиях атмосферного нейтронного воздействия

\begin{tabular}{|c|c|c|c|c|c|c|c|c|}
\hline \multirow[t]{2}{*}{$\begin{array}{l}\text { Технология, } \\
\text { нм / } \\
\text { серия Плис }\end{array}$} & \multicolumn{2}{|c|}{$\begin{array}{c}\text { LANSCE, } \\
\text { сечение взаимодей- } \\
\text { ствия в расчете на бит }\end{array}$} & \multicolumn{2}{|c|}{$\begin{array}{c}\lambda, \\
\text { ФИТ / Мбайт } \\
\text { (тепловые нейтроны) }\end{array}$} & \multicolumn{2}{|c|}{$\begin{array}{c}\lambda, \\
\text { ФИТ / Мбайт } \\
\text { (альфа-частицы) }\end{array}$} & \multicolumn{2}{|c|}{$\begin{array}{c}\lambda \text { в реальном } \\
\text { времени, } \\
\text { ФИТ / Мбайт }\end{array}$} \\
\hline & CRAM & Ошибки, \% & CRAM & Ошибки, \% & CRAM & Ошибки, \% & CRAM & Ошибки, \% \\
\hline $\begin{array}{l}180 \text { нм / } \\
\text { Virtex-E }\end{array}$ & $1,12 \cdot 10^{-14}$ & \pm 18 & - & - & - & - & 181 & \pm 20 \\
\hline $\begin{array}{l}65 \text { нм / } \\
\text { Virtex-5 }\end{array}$ & $6,70 \cdot 10^{-15}$ & \pm 18 & - & - & - & - & 165 & $-13,+15$ \\
\hline $\begin{array}{l}45 \text { нм / } \\
\text { Spartan-6 }\end{array}$ & $1,00 \cdot 10^{-14}$ & \pm 18 & 21 & $-11,+13$ & 88 & $-50,+100$ & 177 & $-10,+11$ \\
\hline $\begin{array}{l}28 \text { нм / } \\
\text { Kintex-7, } \\
\text { Virtex-7 }\end{array}$ & $5,69 \cdot 10^{-15}$ & \pm 18 & 1,1 & $-15,+18$ & 43 & $-41,+80$ & 50 & $-20,+26$ \\
\hline $\begin{array}{l}16 \text { нм / } \\
\text { UltraScale+ }\end{array}$ & $6,67 \cdot 10^{-16}$ & \pm 18 & 0,35 & $-16,+20$ & 0,1 & $-20,+20$ & 5 & $-27,+39$ \\
\hline
\end{tabular}




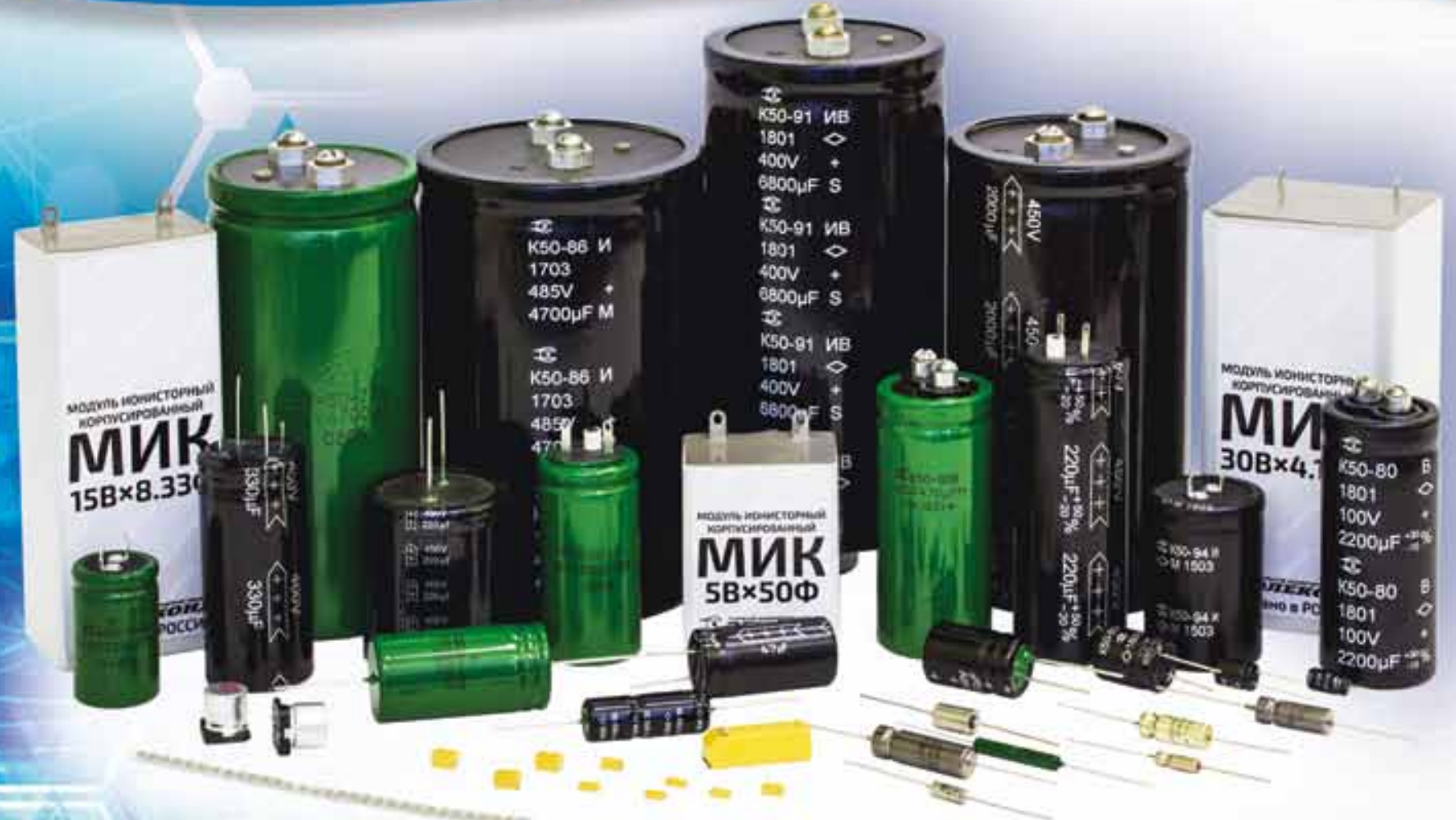

\section{Разработка и производство конденсаторов}

оксидно-электролитические алюминиевые конденсаторы К50-15, К50-17, К50-27, К50-37, К50-68, К50-74, К50-76, К50-77, К50-80, К50-81, К50-83, К50-84, К50-85, К50-86, К50-87, К50-88, К50-89, К50-90, К50-91, К50-92, К50-93, К50-94, К50-95(чип), К50-96, К50-98

объемно-пористые танталовые конденсаторы К52-1, К52-1М, К52-1БМ, К52-1Б, К52-9, К52-11, К52-17, К52-18, К52-19, К52-20, К52-21, К52-24, К52-26(чип), К52-27(чип)

оксидно-полупроводниковые танталовые конденсаторы

К53-1А, К53-7, К53-65(чип), К53-66, К53-68(чип), К53-71(чип), К53-72(чип), К53-74(чип), К53-77(чип), К53-78(чип)

суперконденсаторы (ионисторы) K58-26

накопители электрической энергии на основе модульной сборки суперконденсаторов

Система менеджмента качества сертифицирована на соответствие требованиям ISO 9001

Россия, 427968, Удмуртская Республика, г. Сарапул, ул. Калинина, 3 Тел.: (34147) 2-99-53, 2-99-89, 2-99-77, факс: (34147) 4-32-48, 4-27-53 e-mail: elecond-market@elcudm.ru, http://www.elecond.ru 
в ФИТах / Мбайт) для ПлИС на базе СОЗУ от Xilinx разных технологических поколений. Эти данные были получены по результатам ускоренных испытаний с использованием источника нейтронов LANSCE (Los Alamos Neutron Science Center, Лос-Аламосский центр по изучению физики нейтронов) и измерений атмосферного нейтронного воздействия, проводимых в реальном времени (на уровне системы) в местностях, различающихся высотой над уровнем моря (горные вершины и под землей), в соответствии с методикой JESD89A / 89-1А [13]. При измерениях учитывались ошибки в ПлиС, вызванные одиночными сбоями ячеек конфигурационной памяти CRAM вследствие воздействия ионизирующего излучения.

При испытаниях использовалась специальная экспериментальная установка LANSCE для имитации радиационной среды земной атмосферы.

Ошибки в Плис оказывают влияние на работу системы, вызывая функциональные прерывания (Single Event Functional Interrupt - SEFI). Для оценки влияния ионизирующего излучения на проекты, реализованные в базисе плис, применяется такое понятие, как "фактор уязвимости устройства" (DVF). DVF для типичного проекта в ПлИС составляет 5\%, поскольку не все конфигурационные биты Плис могут использоваться в проекте и из-за наличия критичных бит для реализуемой логической функции. В наихудшем случае DVF не превышает 10\% сбоев, вызывающих программную ошибку.

Ранние ПлИс Xilinx серии Virtex-II демонстрировали интенсивность программных ошибок на уровне 405 ФИТ / Мбайт и содержали около 106 конфигурационных бит. Данные, представленные в табл. 4, показывают, что для ПЛИС, выпущенных по 45-, 28- и 16-нм проектным нормам, характерно устойчивое снижение интенсивности программных ошибок как в условиях воздействия тепловых нейтронов и альфа-частиц при экспериментальных испытаниях с использованием источника нейтронов LANSCE, так и при атмосферном нейтронном воздействии. Непрерывное снижение интенсивности программных ошибок стало возможным в результате применения инновационных конструкторскотехнологических решений, например при разработке ячейки памяти, обладающей иммунитетом к SEU.

\section{ЗАКЛЮЧЕНИЕ}

Для оценки экспериментальной интенсивности отказов ПлиС по результатам квалификационных испытаний в производственных условиях используется статистика $\chi^{2}$, которая справедлива только для периода нормальной работы ИС на "ваннообразной" кривой интенсивности отказов, когда "ранние» отказы исключены, а также при больших значениях эквивалентных приборо-часов (EDH). Результаты ускоренных испытаний (ускоряющий фактор - температура в сочетании с повышенным напряжением), проведенных зарубежными компаниями-производителями, показали сверхнизкую интенсивность отказов Плис, не превышающую 200 ФИТ, что гарантирует 100 тыс. ч безотказной работы.

По мере уменьшения минимальных геометрических размеров элементов и снижения напряжения питания современных ПлИС сбои, вызванные ионизирующим излучением, становятся все более важным фактором, влияющим на характеристики ИС. Для снижения интенсивности сбоев компании внедряют как схемотехнические, так и технологические решения, направленные на повышение стойкости Плис к внешним воздействиям.

\section{ЛИТЕРАТУРА}

1. Строгонов А. Оценка долговечности БИС по результатам ускоренных испытаний // Технологии в электронной промышленности. 2007. № 3. С. 10-16.

2. Строгонов А., Цыбин С., Городков П. Расчет количественных показателей надежности цифровых БИС с использованием справочника MIL-HDBK-217F и программы MTBF Calculator фирмы ALD // Компоненты и технологии. 2015. № 1. С. 6-12.

3. Silicon Solution Company. Oki Electric Industry Co., Ltd. '04 Hand Book for QUALITY/RELIABILITY. May 11, 2004.

4. ADI Reliability Handbook. monitoring_predication.pdf. http://www.analog.com

5. http://www.analog.com/media/en/technicaldocumentation/user-guides/UG-311.pdf

6. https://www.onsemi.com/pub/Collateral/HBD851-D.PDF. Quality \& Reliability Handbook

7. 2010 Atmel Corporation. Rev.: ATMELQHBK http://citeseerx.ist.psu.edu/viewdoc/download?doi=10.1.1 188.4679\&rep=repl\&type=pdfAtmel Quality Handbook

8. Reliability Report 57. IH 2014. - www.altera.com

9. Reliability Report. 1H 2017. - www.intel.com

10. Quality \& Reliability Guide. February 2001. - www.actel. com

11. Reliability Report. RT0001. Microsemi FPGA and SoC Products. - www.microsemi.com

12. Device Reliability Report. Second Half 2017. UG116 (v10.8). March 22, 2018. - www.xilinx.com

13. Device Reliability Report. Second Half 2018. UG116 (v10.10). March 22, 2019. - www.xilinx.com

14. плис и параллельные архитектуры для применения в аэрокосмической области. Программные ошибки и отказоустойчивое проектирование / Под ред. Кастеншмидт Ф., Реха П.; перевод с англ. и научная редакция Цыбина С.А, Быстрицкого А.В, Строгонова А. В., Городкова П. С. - М.: ТЕХНОСФЕРА, 2018. 326 С. 